\title{
DIVERSIDAD Y ABUNDANCIA DE COMUNIDADES ZOOPLANCTONICAS LITORALES DEL HUMEDAL PANTANOS DE VILLA, LIMA, PERU
}

\author{
DIVERSITY AND ABUNDANCE OF LITTORAL ZOOPLANKTONIC \\ COMMUNITIES OF PANTANOS DE VILLA WETLAND, LIMA, PERU
}

\author{
José Iannacone ${ }^{1,2} \&$ Lorena Alvariño ${ }^{2}$ \\ ${ }^{1}$ Laboratorio de Invertebrados. Facultad de Ciencias Biológicas. Universidad Ricardo Palma, Lima, Perú. \\ Email: joseiannacone@yahoo.es \\ ${ }^{2}$ Laboratorio de Ecofisiología Animal. Facultad de Ciencias Naturales y Matemática. Universidad Nacional \\ Federico Villarreal. Lima, Perú. Email: lalvarino@sedapal.com.pe
}

\section{RESUMEN}

Pantanos de Villa es un humedal costero conformado por varios cuerpos de agua, permanentes y estacionales. Este ecosistema es reconocido internacionalmente como sitio RAMSAR y se encuentra ubicado al sur de la zona urbana de Lima, Perú. En junio y octubre de 2004 y en abril del 2005 se llevaron a cabo censos de la biodiversidad y abundancia de las comunidades zooplanctónicas litorales, representando a diferentes cuerpos de agua permanentes en un total de 21 puntos de muestreo. Las muestras de zooplancton fueron colectadas usando una red de plancton de $75 \mathrm{~mm}$ de diámetro y una apertura de 120 $\mathrm{mm}$, y fueron preservadas empleando una solución de formol azucarado al $4 \%$. Se determinaron algunos parámetros limnológicos como Oxígeno Disuelto $(\mathrm{OD})\left(\mathrm{mg} \mathrm{L}^{-1}\right)$, transparencia $(\mathrm{cm})$, temperatura del agua $\left(\mathrm{T}_{\text {agua }}\left({ }^{\circ} \mathrm{C}\right)\right.$, temperatura del aire $\left(\mathrm{T}_{\text {aire }}\right)\left({ }^{\circ} \mathrm{C}\right)$ y profundidad máxima $(\mathrm{cm})$ de cada cuerpo de agua. Los resultados indicaron la presencia de 43 taxa, siendo más abundantes y frecuentes los rotíferos Brachionus calyciflorus (Pallas 1776) y Epiphanes sensa (Müller 1773), los ostrácodos, el nemátodo Rhabdolaimus terrestris de Mann 1880 y el ciliado Vorticella campanula Ehrenberg, 1831. El número promedio de organismos $\cdot \mathrm{L}^{-1}$ fue de $337 \pm 80 \mathrm{y}$ el número promedio de taxa por punto de muestreo fue de $11,2 \pm 2,1$. Existieron diferencias significativas en el $\mathrm{OD}, \mathrm{T}_{\text {agua }}$ y $\mathrm{T}_{\text {aire }}$ entre las estaciones muestreadas. El análisis de componentes principales (ACP) indicó un componente conformado por $\mathrm{OD}, \mathrm{T}_{\text {agua }}$ y $\mathrm{T}_{\text {aire }} \mathrm{y}$ otro formado por la transparencia y la profundidad. Finalmente, se realizó la valoración de la diversidad alfa y beta de las comunidades zooplanctónicas de este ecosistema acuático estudiado con el fin de proponer medidas para su conservación.

Palabras Claves: Cladóceros, copépodos, diversidad alfa, diversidad beta, limnología, rotíferos.

\section{ABSTRACT}

Pantanos de Villa, is a coastal wetland conformed by several water bodies, permanent and seasonal. This ecosystem is internationally recognized as a RAMSAR site located in the south of the urban zone of Lima, Peru. In June 2004, October 2004 and April 2005, surveys of biodiversity and abundance of littoral zooplanktonic communities, representing to different permanent water bodies in 21 points of sampling in total were carried out. The samples of zooplancton were collected using a $75 \mu \mathrm{m}$ of diameter plankton net and $120 \mathrm{~mm}$ of opening. The samples were preserved and fixed employing a solution of $4 \%$ sweetened formalin. Some limnological parameters as dissolved oxygen (DO) (mg L $\mathrm{L}^{-1}$ ), transparency $(\mathrm{cm})$, water temperature $\left(\mathrm{T}_{\text {water }}\right)\left({ }^{\circ} \mathrm{C}\right)$, air temperature $\left(\mathrm{T}_{\text {air }}\right)\left({ }^{\circ} \mathrm{C}\right)$ and maximum depth of each water body were determined. The results indicated the presence of 43 taxa, being abundant and frequent rotifers Brachionus calyciflorus (Pallas 1776) and Epiphanes sensa (Müller 1773), ostracoda, nematoda Rhabdolaimus terrestris de Mann, 1880 and the ciliate Vorticella campanula Ehrenberg, 1831. Number of organism $\cdot L^{-1}$ was $337 \pm 80$ and number of taxa by point of survey was 11.2 \pm 2 .1. Significantly differences in DO, $\mathrm{T}_{\text {water }}$ and $\mathrm{T}_{\text {air }}$ between stations surveyed were found. Principal Component Analysis (PCA) indicated a component conformed by DO, $\mathrm{T}_{\text {water }}$ and $\mathrm{T}_{\text {air }}$, and other formed by transparency and maximum depth. Finally, a valoration of alpha and beta diversity of zooplanktonic communities was performed in this aquatic ecosystem studied with the aim to propose guidelines for conservation.

KEYwords: Cladocers, copepods, alpha diversity, beta diversity, limnology, rotifer. 


\section{INTRODUCCION}

El ecosistema de los Pantanos de Villa, Lima, Perú, es considerado actualmente un área protegida por el Estado, siendo un humedal de importancia internacional por ser un sitio RAMSAR (Vivar et al. 1998). Este ambiente presenta una gran diversidad de especies de flora y fauna, diversidad paisajística y constituye parte de un corredor ecológico en la ruta migratoria de muchas especies de aves (León et al. 1995, Valqui 2004).

La conservación de esta área natural protegida rodeada por comunidades humanas está amenazada por la destrucción del hábitat por disturbios antrópicos, debido al efecto de la ampliación de la frontera urbana, por la contaminación producida por residuos urbanos que son arrojados en los alrededores de los pantanos, por alteraciones en la calidad del agua por desagues de las empresas agropecuarias e industriales, por perturbaciones por ruido del tránsito vehicular, y probablemente por exceso de visitantes en determinados días de la semana (Young 1998).

La flora representativa de los Pantanos de Villa consiste en 67 especies de plantas en asociaciones características: gramadal en suelos arenosos, cuya especie predominante es la grama salada (Distichlis spicata (Linnaeus) Greene, Poaceae); totoral en áreas inundadas y bordes de los espejos de agua, siendo la especie predominante la totora (Typha dominguensis (Persoon, 1807), Typhaceae); zona arbustiva, que se caracteriza por la apariencia de un matorral denso; y vega de ciperáceas en suelos saturados caracterizada por la presencia de Scirpus americanus (Persoon, 1810) (Cyperaceae) y Paspalum vaginatum Swartz, 1788 (Poaceae). La ornitofauna está representada principalmente por 155 especies de aves, de las cuales 77 son migratorias (Valqui 2004).

En los Pantanos de Villa se han evaluado diversos grupos biológicos de importancia como algas con 49 Bacillariophyceae, 40 Cyanophyceae y 30 Chlorophyceae (Montoya 1984), protozoarios como Mastigophora con 44 especies, Sarcodina con 29 especies y Ciliophora con 98 especies (Sarmiento \& Morales 1998, Guillén et al. 2003), helmintos parásitos como Notocotylus pacifera (Noble, 1939) Harwood, 1939 (Trematoda), Psilostomum sp. (Trematoda) y Diorchis americana Ransom, 1909 (Cestoda) en Fulica ardesiaca Tschudi 1843
(Guillen \& Morales 2003), moluscos como Physa venustula (Gould 1847), Drepanotrema kermatoides (Orbigny, 1835) y Ancylus concentricus (Orbigny 1835) (Vivar et al. 1998), arañas como Tetragnathidae, Araneidae, Sicariidae y Palpimanidae (Duárez 1998), insectos acuáticos, principalmente Belostomatidae, Libellulidae, Dytiscidae e Hydrophilidae (García et al. 1996), y aves como Podicipedidae, Pelecanidae, Sulidae, Ardeidae, Anatidae, Cathartidae, Falconidae, Rallidae, Charadriidae, Scolopacidae, Columbidae, Cuculidae, Strigidae, Furnariidae, Tyraniidae, Troglodytidae, Ploceidae y Fringillidae (Guillen \& Barrio 1995, Valqui 2004), entre otros.

Sin embargo, a pesar que los organismos zooplanctónicos son utilizados para monitorear la calidad del agua (Modenutti 1998, Riofrio 2002, Ramos 2004, Rejas et al. 2005), éstos han sido pobremente evaluados en los Pantanos de Villa (Grosspietsh 1999).

La composición y abundancia de las comunidades zooplanctónicas puede ser influenciada por numerosos factores físicos, químicos y biológicos (Bini et al. 2001, Bledzki \& Ellison 2003, Rejas et al. 2005, Suárez-Morales et al. 2005). La temperatura, el tamaño de los cuerpos de agua, el estado trófico, el estado sucesional, la calidad y la disponibilidad de alimento, la competencia y la depredación pueden afectar tanto la composición como la densidad poblacional del zooplancton, y ser factores que actúen en simultáneo en los ambientes naturales (Rodríguez \& Matsumura-Tundisi 2000, Sampaio et al. 2002, Arora \& Mehra 2003, Nagorskaya 2004, De Meester et al. 2005).

Los principales grupos componentes de las comunidades zooplantónicas son los protozoos, rotíferos y crustáceos, particularmente copépodos y cladóceros. Sin embargo, el zooplancton dulceacuícola, especialmente en las regiones tropicales, puede contener una amplia diversidad de categorías taxonómicas (Santos-Wisniewski et al. 2002, Aoyagui et al. 2003, Suárez-Morales et al. 2004). A la fecha, en los Pantanos de Villa, no se ha evaluado la comunidad zooplanctónica representativa de este humedal, ni se ha empleado índices de diversidad alfa comparativamente.

El objetivo del presente trabajo fue evaluar comparativamente la biodiversidad y abundancia de la comunidad zooplanctónica litoral de los Pantanos de Villa durante el 2004 y el 2005. 


\section{MATERIALES Y METODOS}

Area de estudio

Los Pantanos de Villa están ubicados en el distrito de Chorrillos, al sur del Departamento de Lima, Perú $\left(12^{\circ} 11^{\prime} 42^{\prime \prime}\right.$ - $12^{\circ} 13^{\prime} 18^{\prime \prime}$ LS y $76^{\circ} 58^{\prime} 42^{\prime}$ ' - $76^{\circ}$ 59'42' ' LW). Es la única área protegida que existe dentro del casco urbano de la ciudad de Lima. La extensión actual de esta área protegida es de 276 ha (Resolución Ministerial N 0909-2001) (Amaya et al. 1999). Esta área tiene escasa precipitación durante todo el año y los vientos que se presentan en la zona van de sur a sur-este, los cuales junto con la Corriente Peruana producen una niebla característica. La temperatura durante el verano oscila entre los 15 y $26^{\circ} \mathrm{C}$ y durante el invierno entre los 14 y $19^{\circ} \mathrm{C}$.

El presente estudio se realizó durante tres ocasiones: 2 de junio del 2004 (ocho puntos), 28 de octubre del 2004 (cuatro puntos) y 8 abril del 2005 (nue- ve puntos) (Fig. 1). Para la selección de los puntos de muestreo se tomó en cuenta las recomendaciones de Valdivia \& Zambrano (1989) y Alvariño (1990). Por lo que buscó representar la heterogeneidad espacial de diferentes cuerpos de agua permanentes litorales. Lo cual incluyó lagunas, charcas permanentes y canales de poca profundidad (Young 1998). Las principales características de los puntos de muestreo fueron: Laguna mayor (puntos 2, 3, 5, 10, 13 y 16), la cual presenta una extensión de 50.000 mî y un sustrato arenoso-fangoso; Laguna Génesis (puntos $1,9,11$ y 14), la cual presenta sustrato arenoso fangoso; Laguna Marvilla (puntos 7, 8, 12, 18, 19, 20 y 21), con una extensión de $3.360 \mathrm{~m}^{2}$ y un sustrato tipo arenoso-limoso; charca permanente (puntos 6 y 15) con sustrato arenoso-fangoso, y finalmente un canal de poca profundidad con sustrato tipo arenoso (puntos 4 y 17) (Fig. 1). La hora de colecta se realizó entre las 9:30 a 12:30 h.

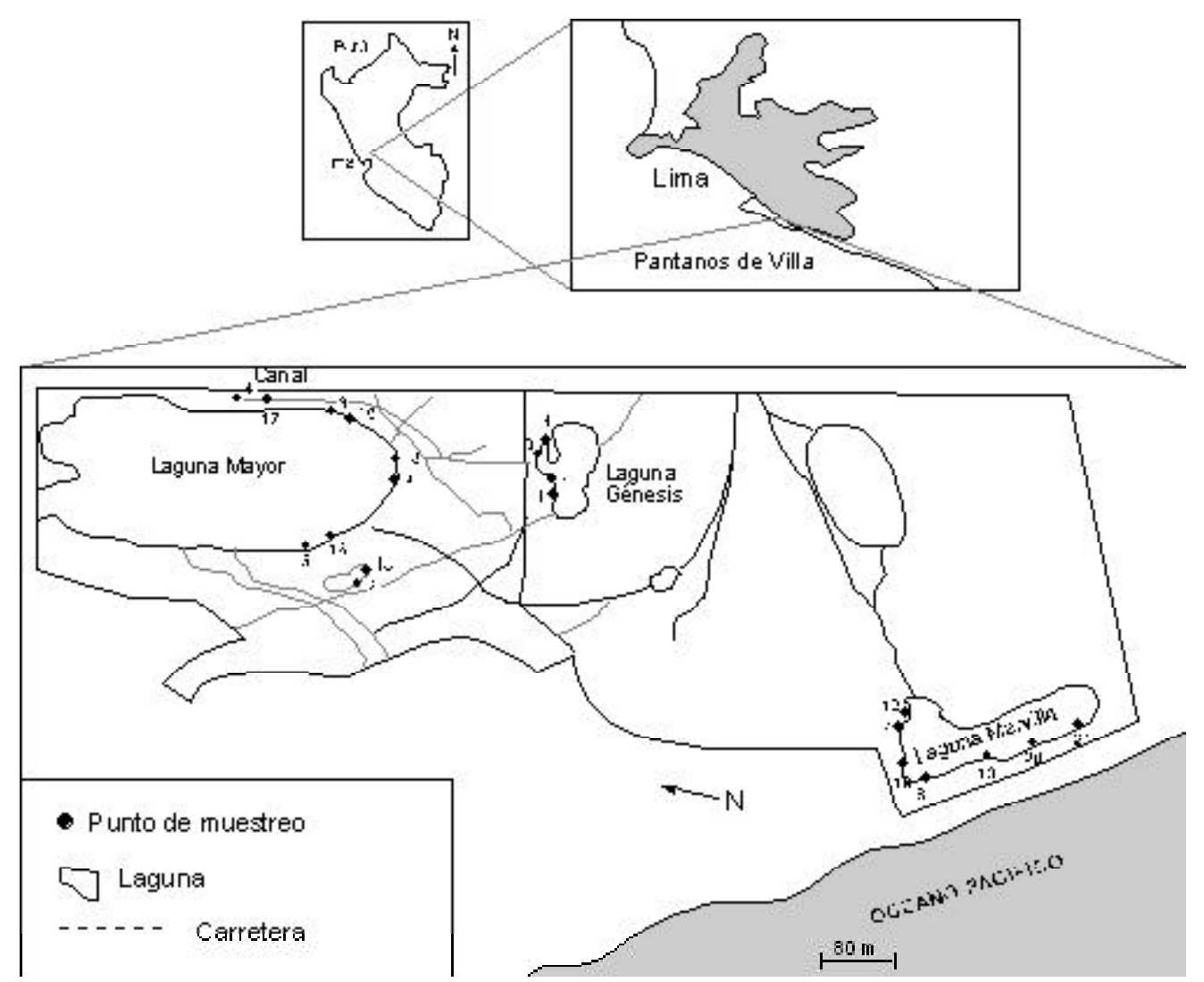

FIGURA 1. Mapa mostrando el área de estudio y los 21 puntos de muestreo.

FIGURE 1. Map showing the studied area and 21 points of sampling. 


\section{ZOOPLANCTONLITORAL}

En cada punto de muestreo se procedió a filtrar el agua, con la ayuda de un balde plástico de $4 \mathrm{~L}$ de capacidad y empleando una red manual de malla Nytal® de 75 $\mathrm{mm}$ de abertura de poro y $120 \mathrm{~mm}$ de diámetro. $\mathrm{El}$ volumen filtrado varió en dependencia a las características de cada punto entre 8 a 20 L (Keppeler \& Hardy 2004a). El zooplancton concentrado en las redes fue vertido a frascos de boca ancha de $200 \mathrm{~mL}$ y posteriormente se les adicionó formol azucarado al $10 \%$ para lograr una concentración de fijación y preservación al 4 \% (Valdivia \& Zambrano 1989), luego fueron etiquetados y transportados al laboratorio para su posterior conteo e identificación taxonómica.

En el análisis cuantitativo se procedió a medir el volumen total de la muestra en una probeta de $100 \mathrm{~mL}$, luego se tomaron submuestras de $3 \mathrm{~mL}$ y se colocaron en una placa Petri de plástico de $60 \mathrm{~mm}$ de diámetro para su observación al microscopio a 400 X y 1000 X de aumento, procediéndose luego al conteo de los especímenes girando la placa en zigzag (Arora \& Mehra 2003). La densidad o abundancia de organismos zooplanctónicos se presentó en $\mathrm{N}^{\circ}$ organismos . $\mathrm{L}^{-1}$ (APHA 1995).

Para la identificación de los especímenes se utilizaron los trabajos sobre cladóceros del Perú y de copépodos de vida libre en el Perú (Valdivia \& Zambrano 1989, Del Río \& Valdivia 1989, Alvariño 1990, Valdivia \& Burger 1990). Para la identificación de los rotíferos se empleó a Koste $(1972,1978)$, a José de Paggi (1989) y a Segers (1995). Para los moluscos se siguió a Vivar et al. (1994), Thiengo et al. (2001) y a Paraense (2003). Para los copépodos se usó a Holynska et al. (2003), a Mirabdullayev et al. (2003) y a Suárez-Morales et al. (2004, 2005). Para el resto de grupos se empleó literatura especializada para su identificación.

Con el objetivo de verificar la constancia de cada taxón en las diferentes estaciones de muestreo fue determinado el índice de constancia (Sampaio et al. 2002). Los taxa fueron considerados constantes (C) cuando fueron registrados en más del 50\% de las muestras, comunes (c) cuando se presentaron entre un 10 y $50 \%$, y finalmente raros (r) hasta en un $10 \%$ de las muestras.

Para el análisis de la dominancia y abundancia de taxa, el criterio propuesto por Lobo \& Leighton (1986) fue aplicado: un taxón fue considerado abundante cuando el número de individuos fue más alto que la densidad promedio de todos los taxa ocurrentes, y domi- nante cuando su densidad numérica fue más alta que el $50 \%$ del total del número de individuos presentes.

VARIABLES FÍSICAS Y QUÍMICAS

Los análisis físicos y químicos incluyeron mediciones de temperatura del agua y del aire en ${ }^{\circ} \mathrm{C}$ mediante un termómetro de mercurio. El oxígeno disuelto (OD) en $\mathrm{mg} \mathrm{L}^{-1}$ siguió el protocolo propuesto por Winkler y la transparencia del agua en cm empleó el Disco de Secchi (APHA, 1995; Wetzel \& Likens 2001). La profundidad máxima por punto de muestreo en $\mathrm{cm}$ se midió con la ayuda de una varilla de madera calibrada en cm (Iannacone et al. 2003). Solo en el muestreo de abril del 2005 se incluyó el pH y la conductividad en Ohms $\cdot \mathrm{cm}^{-1}$.

\section{AnÁLisisde DATOS}

Se empleó el coeficiente de correlación Spearman $\left(\mathrm{r}_{\mathrm{s}}\right)$ para determinar la relación entre las abundancias de taxa del zooplancton $\left(\mathrm{N}^{\circ}\right.$ organismos $\left.\mathrm{L}^{-1}\right)$ entre las tres ocasiones evaluadas. En adición, se usó para relacionar entre la riqueza del número de taxa nuevos totales: de rotíferos, de cladóceros y de protistas. Nuevamente se empleó, para determinar la relación entre la riqueza del número de taxa totales, de rotíferos, de cladóceros, de copépodos y de protista, entre los 21 puntos de muestreos.

Se emplearon tres métodos no paramétricos: Chao-2, Jacknife de primer orden y Jacknife de segundo orden para estimar el número máximo de riqueza de taxa de zooplancton total, de rotíferos, de cladóceros y de protistas a partir del esfuerzo de muestreo realizado y de la acumulación de nuevos taxa que van apareciendo en las muestras (Moreno 2001).

Se empleó el análisis de componentes principales (ACP) como un criterio de reducción y ordenación de los cinco parámetros físicos y químicos y de los diez índices de diversidad alfa, obtenidos de cada una de las 21 estaciones de muestreo durante el 2004 y 2005 , para producir variables compuestas no relacionadas. La rotación varimax fue realizada para facilitar la interpretación de los componentes del ACP (Zar 1996, Vivanco 1999).

Para el cálculo de la diversidad ecológica alfa se emplearon comparativamente varios índices para dar mayor robustez a los resultados: el índice de ShannonWiener (H') (bits·ind), el índice de Simpson (C), el índice de Margalef $\left(\mathrm{D}_{\mathrm{mg}}\right)$, el índice de equidad de Pielou ( $\mathrm{J}^{\prime}$ ), el índice de dominancia de McIntosh (D), el Indice de Menhinick $\left(\mathrm{D}_{\mathrm{Mn}}\right)$ y el índice de Berger- 
Parker (d) (Moreno 2001). Para el análisis de la diversidad ecológica beta comparativo de similaridad entre las tres localidades se aplicaron los índices cualitativos de Sörensen $\left(\mathrm{I}_{\mathrm{S}}\right)$, de Jaccard $\left(\mathrm{I}_{\mathrm{J}}\right)$, de Sokal y Sneath $\left(\mathrm{I}_{\mathrm{S}-\mathrm{S}}\right)$, y de Ochiai- Barkman $\left(\mathrm{I}_{\mathrm{O}-\mathrm{B}}\right)$, así como los índices cuantitativos de Sörensen (Bray-Curtis)( Is $_{\text {cuantitativo }}$ y de MorisitaHorn $\left(\mathrm{I}_{\mathrm{M}-\mathrm{H}}\right)$ (Moreno 2001, Medri \& Lopes 2001, Iannacone et al. 2003). Se usó el coeficiente de correlación de Pearson (rs) para relacionar los seis índices de similaridad entre los tres periodos evaluados. En adición, se determinó el índice beta2 , que mide el grado en el cual la riqueza global de taxa excede a la máxima riqueza muestreal (Harrinson et al. 1992).

El ANDEVA fue usado para determinar si existían diferencias significativas para cada uno de los cinco parámetros físicos y químicos y los diez parámetros de diversidad alfa entre los tres periodos evaluados. En el caso de existir diferencias significativas se aplicó la prueba a posteriori de Tukey (Zar 1996). El nivel de significancia empleado fue de alfa = 0,05 (Zar 1996). Se utilizó el paquete estadístico SPSS versión 13,0 para el cálculo de los estadísticos descriptivos e inferenciales.

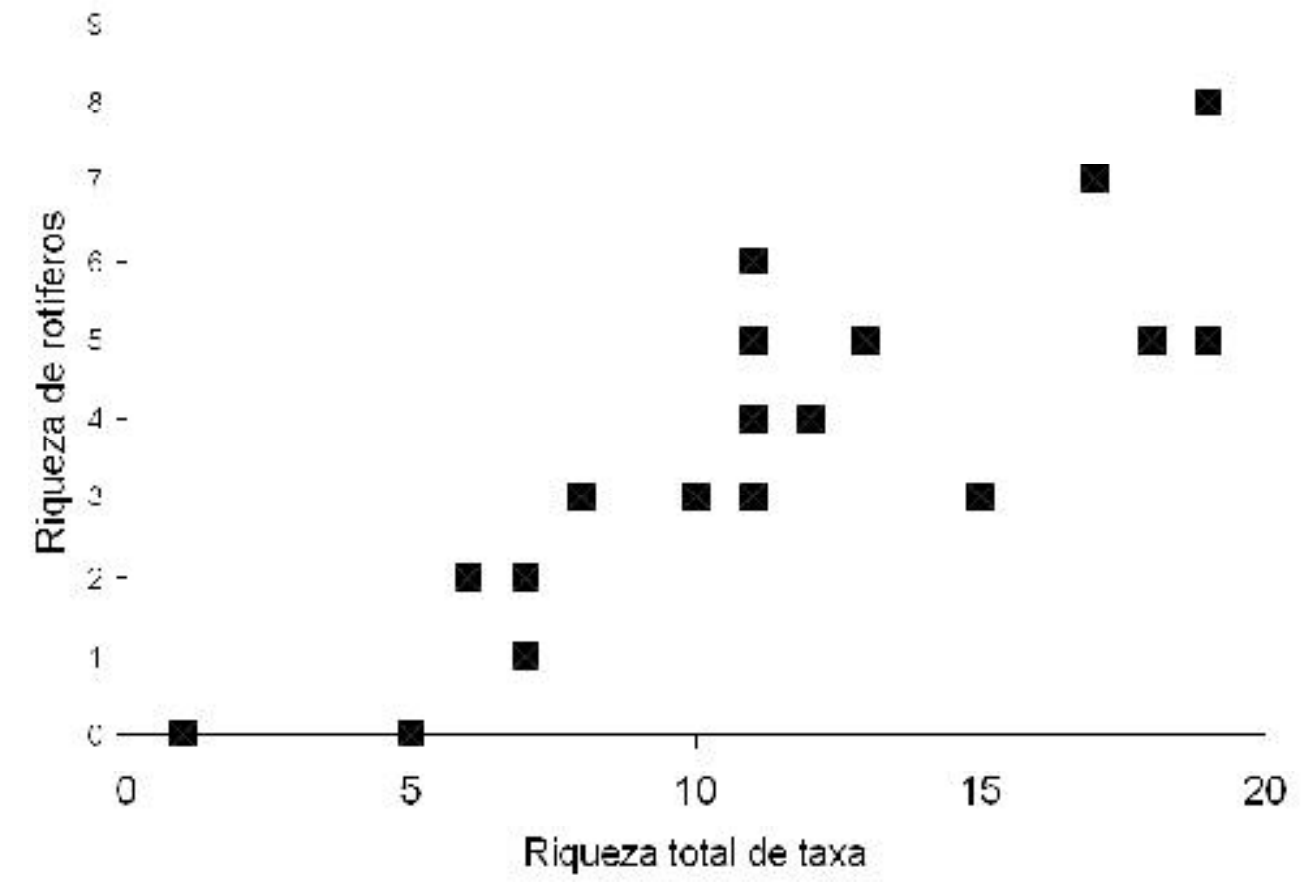

FIGURA 2. Congruencia entre la riqueza total de taxa de las comunidades zooplanctónicas y la riqueza del número de rotíferos.

FIGURE 2. Congruence between total richness of taxa of zooplanktonic communities and total richness of rotifers.

\section{RESULTADOS}

COMPOSICIÓN TAXONÓMICA Y ABUNDANCIA DEL ZOOPLANCTON

Considerando las tres ocasiones de evaluación, un total de 43 taxas de zooplancton fueron registrados, los que consistieron principalmente en 6 taxa de protistas, 17 rotíferos, 7 cladóceros y 3 copépodos (Tabla I). El número promedio de organismos $\cdot \mathrm{L}^{-1}$ fue de $337 \pm 80 \mathrm{y}$ el número promedio de taxa por punto de muestreo fue de 11,2 $\pm 2,1$. En junio de 2004, el zooplancton estuvo compuesto de 4 taxa de protistas, 12 rotíferos, 4 cladóceros y 3 copépodos. En octubre del 2004, el zooplancton 
estuvo compuesto de 2 taxa de protistas, 11 rotíferos, 5 cladóceros y 2 copépodos. En cambio, en abril del 2005, el zooplancton estuvo compuesto de 4 taxa de protistas, 10 rotíferos, 3 cladóceros y 2 copépodos (Tabla I). La mayor riqueza de taxa zooplanctónicas se encontró en el muestreo de junio del 2004, seguido por el muestreo de octubre de 2004 y abril de 2005. Se observó una correlación positiva entre la riqueza total de taxa y la riqueza de rotíferos $(\mathrm{r}=0,85 ; \mathrm{P}<0,001)$ (Fig. 2); entre la riqueza total de taxa y la riqueza de copépodos $(\mathrm{r}=0,67 ; \mathrm{P}=0,001)$; entre la riqueza total de taxa $\mathrm{y}$ la riqueza de cladóceros $(\mathrm{r}=0,65 ; \mathrm{P}<0,001)$; ausencia de correlación entre la riqueza total de taxa y la riqueza de protistas $(\mathrm{r}=0,34 ; \mathrm{P}=0,12)$.

Con relación a la abundancia, los rotíferos fueron más abundantes en número de organismos y porcentualmente durante las tres ocasiones de muestreo (Fig. 3a,b). La abundancia decrece en la siguiente secuencia en junio del 2004: rotíferos, protistas, cladóceros y copépodos; en octubre del 2004, abril del 2005 y en el promedio total: rotíferos, protistas, copépodos y cladóceros (Fig $3 a, b)$. Se observa un incremento en la abundancia de rotíferos y una disminución de cladóceros en abril de 2005 (Fig 3a,b).

De los 43 taxa evaluados, seis fueron considerados constantes (en $+50 \%$ de las muestras), 18 comunes (entre 10 y $50 \%$ de las muestras) y 19 raros ( $<10 \%$ de las muestras). Los seis taxa constantes fueron en orden decreciente: Rhabdolaimus terrestris (Nematoda), ostracoda (Crustácea), Vorticella campanula (Protista), Epiphanes sensa (Rotífera), Brachionus calyciflorus (Rotífera) y Mesocyclops longisetus (Copépoda). Ningún cladócero fue considerado especie constante (Tabla I). De los cladóceros, las especies más abundantes fueron Alona combouei y Ceriodaphnia dubia (Tabla I).

9 taxas fueron consideradas abundantes: 1 protista $V$. campanula, 1 nematodo $R$. terrestris, 3 rotíferos E. sensa, B. calyciflorus y B. dimidiatus, 2 cladóceros $A$. combouei y $C$. dubia, 1 copépodo $M$. longisetus y un ostrácodo, con género y especie no identificados (Tabla I). En cambio, ningún taxa fue considerado dominante al no presentar una densidad numérica más alta que el $50 \%$ del total del número de individuos presentes (Tabla I).

TABLA I. Inventario faunístico e Indices de constancia (C) de las taxa pertenecientes a las comunidades zooplanctónicas litorales del humedal Pantanos de Villa, Lima, Perú.

TABLE I. Faunistic inventary and constancy indexes (C) of taxa belonging to littoral zooplanktonic communities of Pantanos de Villa wetland, Lima, Peru.

\begin{tabular}{|c|c|c|c|c|c|}
\hline \multirow[t]{2}{*}{ Taxa zooplanctónicos } & $\begin{array}{l}\text { junio } \\
2004 \\
\end{array}$ & $\begin{array}{l}\text { octubre } \\
2004 \\
\end{array}$ & $\begin{array}{l}\text { abril } \\
2005 \\
\end{array}$ & $\bar{X}$ & \multirow[t]{2}{*}{$\begin{array}{l}N^{\circ} \text { estaciones } \\
\text { que presentó }\end{array}$} \\
\hline & \multicolumn{4}{|c|}{$\mathrm{N}^{\mathrm{o}}$ org $\cdot \mathrm{L}^{-1}$} & \\
\hline Gastotricha & & & & & \\
\hline $\begin{array}{l}\text { Chaetonotus sp. } \\
\text { Nematoda }\end{array}$ & 0 & 2 & 0 & 0,38 & $1 \mathrm{r}$ \\
\hline $\begin{array}{l}\text { Rhabdolaimus terrestris De Mann } 1880 \\
\text { Rotífera }\end{array}$ & 22 & 11 & 31 & 23,76 & $17 \mathrm{C}$ \\
\hline Asplachna priodonta Gosse 1851 & 12 & 2 & 1 & 5,38 & $5 \mathrm{c}$ \\
\hline Brachionus calyciflorus Pallas 1776 & 39 & 22 & 68 & 48,19 & $15 \mathrm{C}$ \\
\hline Brachionus dimidiatus Bryce 1931 & 17 & 3 & 17 & 14,33 & $4 c$ \\
\hline Brachionus spp. (huevos) & 7 & 0 & 0 & 2,67 & $3 \mathrm{c}$ \\
\hline Epiphanes sensa (Müller 1773) & 37 & 26 & 68 & 48,19 & $15 \mathrm{C}$ \\
\hline Euchlanis dilatata (Ehrenberg 1832) & 0 & 3 & 9 & 4,43 & $4 c$ \\
\hline Filinia longiseta (Ehrenberg 1834) & 0 & 2 & 0 & 0,38 & $1 \mathrm{r}$ \\
\hline Hexarthra bulgarica (Wiszniewski 1933) & 0 & 0 & 1 & 0,43 & $1 \mathrm{r}$ \\
\hline Keratella cochlearis (Gosse 1851) & 6 & 0 & 1 & 2,71 & $4 \mathrm{c}$ \\
\hline Keratella tropica (Apstein 1907) & 0 & 1 & 0 & 0,19 & $1 \mathrm{r}$ \\
\hline Lecane leontina (Turner 1892) & 1 & 0 & 0 & 0,38 & $1 \mathrm{r}$ \\
\hline Lecane lunaris (Ehrenberg 1832) & 1 & 3 & 14 & 6,95 & $5 c$ \\
\hline Lecane luna (OFM 1776) & 2 & 0 & 10 & 5,05 & $1 \mathrm{r}$ \\
\hline Lecane ludwigi (Eckstein 1883) & 1 & 0 & 0 & 0,38 & $1 \mathrm{r}$ \\
\hline Lepadella patella (OFM 1786) & 12 & 6 & 0 & 5,71 & $4 c$ \\
\hline
\end{tabular}


Continuación Tabla I.

\begin{tabular}{|c|c|c|c|c|c|}
\hline \multirow[t]{2}{*}{ Taxa zooplanctónicos } & $\begin{array}{l}\text { junio } \\
2004 \\
\end{array}$ & $\begin{array}{c}\text { octubre } \\
2004\end{array}$ & $\begin{array}{r}\text { abril } \\
2005 \\
\end{array}$ & $\bar{X}$ & \multirow[t]{2}{*}{$\begin{array}{l}\mathrm{N}^{\mathrm{o}} \text { estaciones } \\
\text { que presentó }\end{array}$} \\
\hline & \multicolumn{4}{|c|}{$\mathrm{N}^{\mathrm{o}}$ org $\cdot \mathrm{L}^{-1}$} & \\
\hline Philodina acuticornis Murray 1902 & 0 & 3 & 0 & 0,57 & $1 \mathrm{r}$ \\
\hline Ptygura longicornis (Davis 1867) & 1 & 4 & 1 & 1,57 & $3 \mathrm{c}$ \\
\hline \multicolumn{6}{|l|}{ Annelida } \\
\hline Aelosoma variegatum Vejdovsky 1886 & 0 & 1 & 1 & 0,62 & $3 \mathrm{c}$ \\
\hline \multicolumn{6}{|l|}{ Mollusca (Fauna acompañante) } \\
\hline Drepanotrema kermatoides (Orbigny 1835)* & 16 & 0 & 1 & 6,52 & $3 \mathrm{c}$ \\
\hline Heleobia cumingii (Orbigny 1835)* & 1 & 1 & 1 & 1,00 & $6 c$ \\
\hline Physa venustula (Gould 1847)* & 9 & 0 & 0 & 3,43 & $4 \mathrm{c}$ \\
\hline \multicolumn{6}{|l|}{ Cladocera } \\
\hline Alona cambouei Guerne \& Richard 1893 & 29 & 19 & 1 & 15,10 & $10 \mathrm{c}$ \\
\hline Alona rectangula Sars 1861 & 0 & 4 & 0 & 0,76 & $1 \mathrm{r}$ \\
\hline Alonella dentifera Sars 1901 & 0 & 1 & 0 & 0,19 & $1 \mathrm{r}$ \\
\hline Ceriodaphnia dubia Richard, 1894 & 22 & 0 & 0 & 8,38 & $1 \mathrm{r}$ \\
\hline Kurzia latissima (Kurz 1875) & 1 & 1 & 0 & 0,57 & $2 \mathrm{r}$ \\
\hline Moina micrura Kurz, 1874 & 0 & 0 & 1 & 0,43 & $1 \mathrm{r}$ \\
\hline Simocephalus vetulus (Müller 1776) & 1 & 2 & 1 & 1,19 & $4 c$ \\
\hline \multicolumn{6}{|l|}{ Copépoda } \\
\hline Mesocyclops longisetus (Thiébaud, 1914) & 23 & 11 & 12 & 16,00 & $13 \mathrm{C}$ \\
\hline Thermocyclops decipiens (Kiefer 1929) & 1 & 0 & 0 & 0,38 & $1 \mathrm{r}$ \\
\hline Harpacticoidea gen. sp. no identificada & 2 & 1 & 1 & 1,38 & $7 \mathrm{c}$ \\
\hline \multicolumn{6}{|l|}{ Ostracoda } \\
\hline Ostracoda gen. sp. no identificada & 54 & 69 & 5 & 35,86 & $16 \mathrm{C}$ \\
\hline \multicolumn{6}{|l|}{ Acari } \\
\hline Acarina gen. sp. no identificada & 1 & 0 & 1 & 0,81 & $5 \mathrm{c}$ \\
\hline \multicolumn{6}{|l|}{ Insecta (Fauna acompañante) } \\
\hline Plecoptera gen. sp. no identificada* & 0 & 1 & 1 & 0,62 & $2 \mathrm{r}$ \\
\hline Chironomus calligraphus (Goeldi 1905) & 1 & 7 & 3 & 3,00 & $9 \mathrm{c}$ \\
\hline \multicolumn{6}{|l|}{ Protista (Fauna acompañante) } \\
\hline Peridinium $\mathrm{sp}$ & 1 & 0 & 0 & 0,38 & $1 \mathrm{r}$ \\
\hline Arcella vulgaris Ehrenberg, 1830 & 0 & 0 & 2 & 0,86 & $1 \mathrm{r}$ \\
\hline Centropyxis aculeata (Ehrenberg 1832) & 4 & 18 & 5 & 7,10 & $10 \mathrm{c}$ \\
\hline Difflugia lobostoma Leidy 1879 & 0 & 0 & 2 & 0,86 & $1 \mathrm{r}$ \\
\hline Paramecium aurelia (Ehrenberg 1838) & 1 & 0 & 0 & 0,38 & $2 \mathrm{r}$ \\
\hline Vorticella campanula Ehrenberg 1831 & 86 & 23 & 39 & 53,86 & $15 \mathrm{C}$ \\
\hline $\mathrm{N}^{\circ}$ Taxas & 31 & 28 & 28 & 29,14 & \\
\hline
\end{tabular}

$\mathrm{C}=$ Constante $\mathrm{c}=$ común; $\mathrm{r}=$ rara. $*$ = No típicos del zooplancton. $\overline{\mathrm{X}}=$ Promedio ponderado de los 21 muestreos.

VARIABLES FÍSICAS Y QUÍMICAS

La Tabla II nos muestra los valores para las cinco variables fisicoquímicas en los 21 muestreos realizados en junio de 2004, octubre de 2004, abril de 2005 y el valor promedio. La temperatura del agua y del aire, así como el oxígeno disuelto presentaron diferencias estadísticamente significativos entre los tres periodos evaluados. La temperatura del agua fue más baja en junio de $2004\left(5^{\circ} \mathrm{C}\right.$ menos) en comparación con octubre de 2004 y abril de 2005 ( $\mathrm{F}=20,48 ; \mathrm{P}=0,00)$. El mismo patrón se encontró para la temperatura del aire $\left(5^{\circ} \mathrm{C}\right.$ menos $)(\mathrm{F}=16,22 ; \mathrm{P}=0,00)$ y para el oxígeno disuelto $\left(2 \mathrm{mg} \cdot \mathrm{L}^{-1}\right.$ menos $)(\mathrm{F}=4,24 ; \mathrm{P}=0,03)$. Sin embargo, no se encontraron diferencias significativas para la transparencia $(\mathrm{F}=1,91 ; \mathrm{P}=0,17)$ y para la profundidad del cuerpo de agua $(\mathrm{F}=1,55$; $\mathrm{P}=0,23)$. De las 21 muestras analizadas 20 permitieron la transmisión de la luz $(95,2 \%)$ y solo una no $(4,8 \%)$ según el disco de Secchi que evaluó la transparencia del agua. Sólo en abril de 2005 se evaluó el pH siendo 7,9 \pm 0,3 (rango de 7,4 a 8,5), y la conductividad, la cual fue de $6,9 \pm 2,5$ Ohms $\cdot \mathrm{cm}^{-1}$ (rango de 4,7 a 11,9). 
Gayana 71(1), 2007

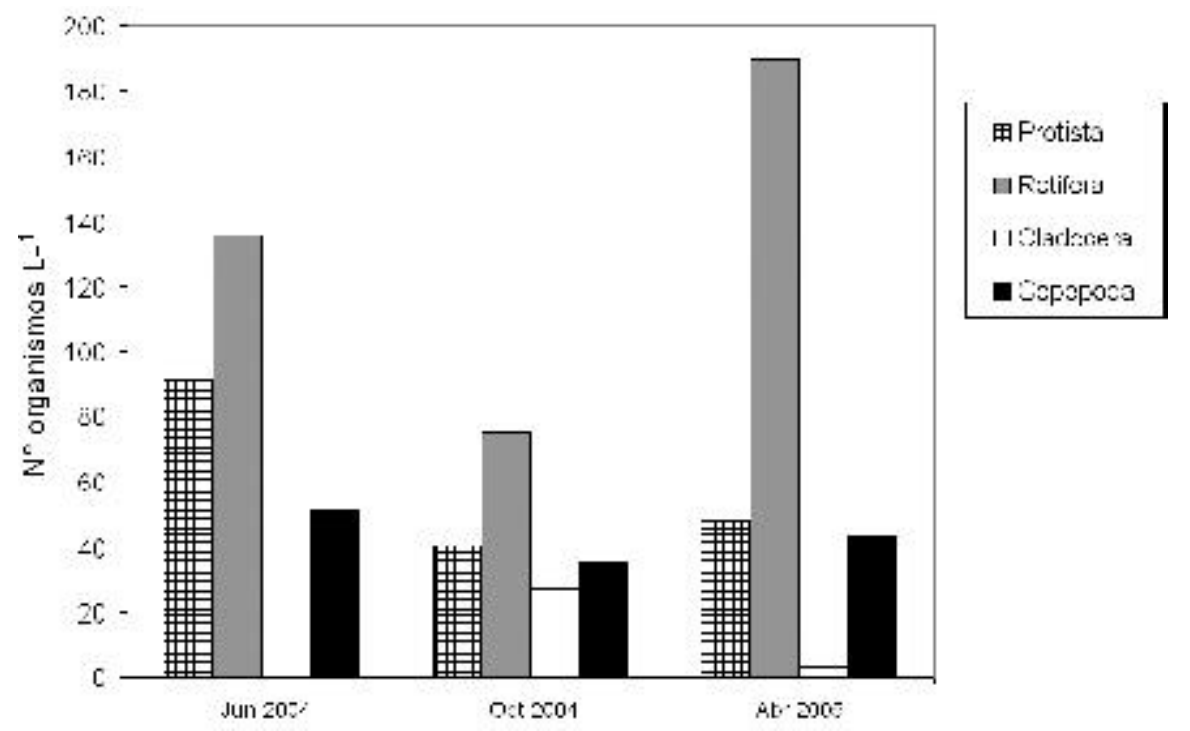

Figura 3a. Cambios en el $\mathrm{N}^{\circ}$ organismos $\mathrm{L}^{-1}$ de las cuatro principales comunidades zooplanctónicas en los Pantanos de Villa, Lima, Perú.

Figure 3a. Changes in the number of organisms $\mathrm{L}^{-1}$ of four main zooplanktonic communities in Pantanos de Villa, Lima, Peru.

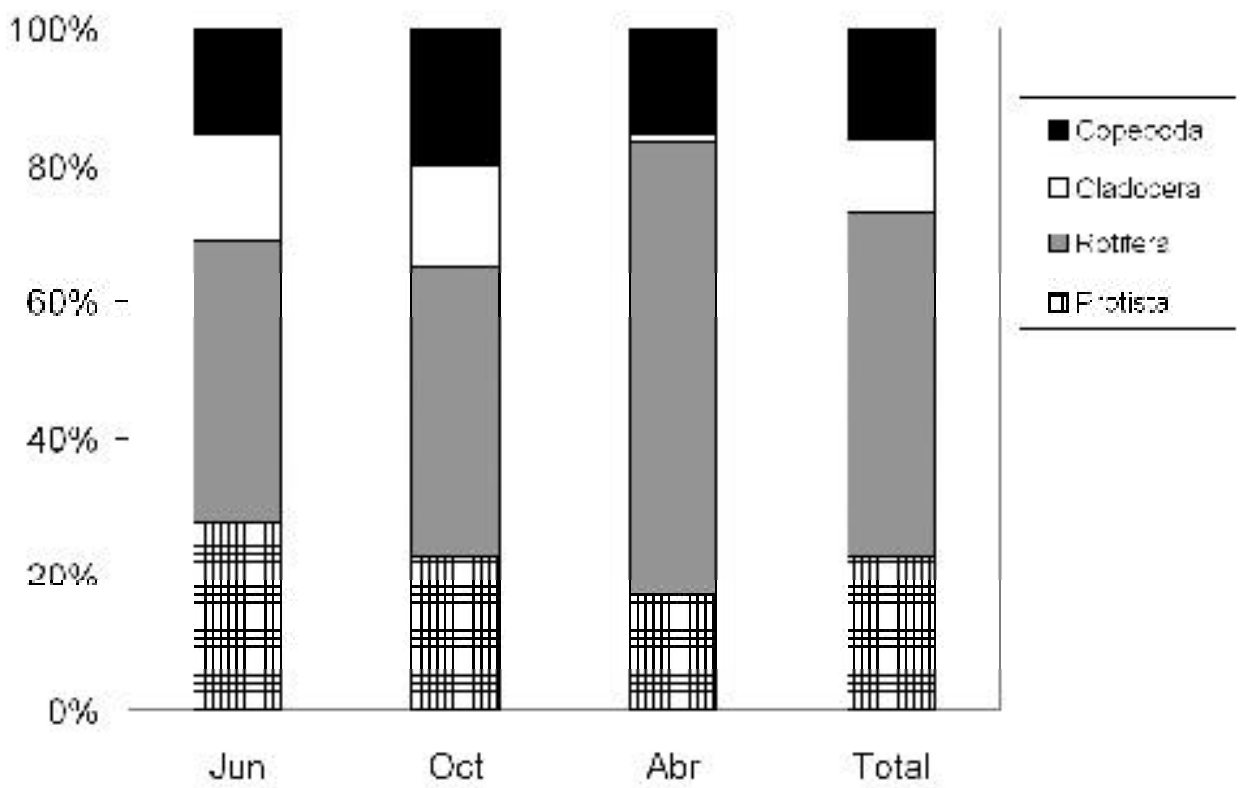

FigURA 3b. Abundancia relativa de las cuatro principales comunidades zooplanctónicas en los Pantanos de Villa, Lima, Perú.

FIgURE 3b. Relative abundance of four main zooplanktonic communities in Pantanos de Villa, Lima, Peru. 
Zooplancton en los Pantanos de Villa, Perú: Iannacone, J. \& L. Alvariño

TABLA II. Parámetros físicos y químicos para Pantanos de Villa.

TABLE II. Physical and chemical parameters for Pantanos de Villa.

\begin{tabular}{lcccc}
\hline Variables & junio & octubre & abril & $\mathrm{X} \pm \mathrm{DE}$ \\
& 2004 & 2004 & 2005 & \\
\hline Temperatura del agua $\left({ }^{\circ} \mathrm{C}\right)$ & $19,8 \pm 1,3 \mathrm{a}$ & $25,3 \pm 1,2 \mathrm{~b}$ & $24,8 \pm 2,4 \mathrm{~b}$ & $23,3 \pm 3,3$ \\
Temperatura del aire $\left({ }^{\circ} \mathrm{C}\right)$ & $17,7 \pm 3,3 \mathrm{a}$ & $23,6 \pm 1,8 \mathrm{~b}$ & $24,7 \pm 2,4 \mathrm{~b}$ & $21,9 \pm 4,2$ \\
OD $\left(\mathrm{mg} \cdot \mathrm{L}^{-1}\right)$ & $2,7 \pm 2,2 \mathrm{a}$ & $5,5 \pm 1,4 \mathrm{~b}$ & $5,2 \pm 2,2 \mathrm{ab}$ & $4,4 \pm 2,4$ \\
Transparencia $(\mathrm{cm})$ & $34,8 \pm 21,9 \mathrm{a}$ & $57,0 \pm 30,4 \mathrm{a}$ & $36,5 \pm 14,7 \mathrm{a}$ & $40,5 \pm 22,4$ \\
Profundidad $(\mathrm{cm})$ & $36,1 \pm 29,5 \mathrm{a}$ & $62,2 \pm 30,9 \mathrm{a}$ & $56,8 \pm 28,8 \mathrm{a}$ & $50,5 \pm 30,3$ \\
\hline
\end{tabular}

$\overline{\mathrm{X}}=$ Promedio. $\mathrm{DE}=$ Desviación estándar.

Letras minúsculas iguales en sentido horizontal indican que los promedios son estadísticamente iguales.

El ACP produjó dos componentes con Eigenvalues > 1 para los parámetros físicos y químicos (Tabla III). Los parámetros físicos y químicos fueron ordenados en un espacio de dos dimensiones, de acuerdo al ACP. La temperatura del agua, del aire y el oxígeno disuelto estuvieron correlacionados con el primer componente, que explicó el 43,477 $\%$ de la variabilidad. La transparencia y la profundidad estuvieron correlacionadas con el segundo componente que explicó el 34,513\% de la variabilidad (Tabla III).

DiVERSIDAD ALFA

No se encontraron diferencias significativas entre las evaluaciones de junio de 2004, octubre del 2004 y abril de 2005 para ninguno de los 10 índices bióticos evaluados (Tabla IV). No se encontraron diferencias significativas para la riqueza específica total $(\mathrm{F}=1,13 ; \mathrm{P}=0,34)$, para la abundancia $(\mathrm{F}=0,12 ; \mathrm{P}=0,88)$, para la riqueza específica de rotíferos $(\mathrm{F}=0,46 ; \mathrm{P}=0,63)$, para Margalef $(\mathrm{F}=0,71 ; \mathrm{P}=0,51)$, para Menhinick $(\mathrm{F}=0,15$; $\mathrm{P}=0,85)$, para Simpson $(\mathrm{F}=0,42 ; \mathrm{P}=0,66)$, para Berger-Parker $(\mathrm{F}=0,38 ; \mathrm{P}=0,69)$, para McIntosh $(\mathrm{F}=0,34 ; \mathrm{P}=0,71)$, para Shannon-Wiener $(\mathrm{F}=0,86$; $\mathrm{P}=0,43)$ y para Pielou $(\mathrm{F}=0,34 ; \mathrm{P}=0,71)$ entre las tres ocasiones de muestreo. El índice de BergerParker y el de Simpson mostraron valores bajos, debido a que las comunidades zooplanctónicas no presentaron dominancia mayor al $50 \%$ de ningún taxa (Tablas I y IV). En cambio los índices de diversidad de Shannon-Wiener y de Pielou fueron relativamente altos para las comunidades zooplanctónicas (Tabla IV).
TABLA III. Resumen del análisis de componentes principales (ACP) de los parámetros fisicoquímicos.

TABLE III. Summary of analysis of principal compounts (APC) for physical and chemical parameters.

\begin{tabular}{lcc}
\hline \multicolumn{1}{c}{ Parámetros } & \multicolumn{1}{c}{ CP1 } & \multicolumn{1}{c}{ CP2 } \\
\hline Eigenvalue & 2,32 & 1,57 \\
\% de la varianza & 43,477 & 34,513 \\
Temperatura del agua & $\mathbf{0 , 9 2 2}$ & 0,032 \\
Temperatura del aire & $\mathbf{0 , 9 1 4}$ & 0,012 \\
OD & $\mathbf{0 , 6 7 6}$ & 0,165 \\
Transparencia & $-0,011$ & $\mathbf{0 , 9 4 8}$ \\
Profundidad & 0,177 & $\mathbf{0 , 8 9 4}$ \\
\hline
\end{tabular}

Los valores más altos en cada componente para cada variable son mostrados en negrita.

El ACP produjo tres componentes con Eigenvalues $>1$ para los índices bióticos (Tabla V). Cinco índices bióticos (Pielou, Berger-Parker, Simpson, McIntosh y Shannon-Wiener) estuvieron correlacionados con el primer componente, que explicó el 45,587 \% de la variabilidad. Otros tres índices bióticos (Riqueza específica de rotíferos, Riqueza específica total y Margalef) estuvieron correlacionados con el segundo componente, que explicó el 29,221\% de la variabilidad (Tabla V). El índice de Menhinick y el $\mathrm{N}^{\circ}$ de organismos $\mathrm{L}^{-1}$ estuvieron relacionados con el tercer componente, que explicó el 19,383 de la variabilidad (Tabla V). 
Gayana 71(1), 2007

TABLA IV. Indices bióticos de diversidad alfa $(\alpha)$ de Pantanos de Villa.

TABLE IV. Biotic indexes of alpha diversity for Pantanos de Villa.

\begin{tabular}{lcccc}
\hline & junio & octubre & abril & $\overline{\mathrm{X}} \pm \mathrm{DE}$ \\
Indices & 2004 & 2004 & 2005 & \\
\hline Pielou, J' & $0,80 \pm 0,1^{\mathrm{a}}$ & $0,84 \pm 0,1^{\mathrm{a}}$ & $0,74 \pm 0,3^{\mathrm{a}}$ & $0,78 \pm 0,2$ \\
Berger-Parker, d & $0,37 \pm 0,1^{\mathrm{a}}$ & $0,27 \pm 0,2^{\mathrm{a}}$ & $0,36 \pm 0,2^{\mathrm{a}}$ & $0,35 \pm 0,2$ \\
Simpson, C & $0,22 \pm 0,1^{\mathrm{a}}$ & $0,17 \pm 0,1^{\mathrm{a}}$ & $0,28 \pm 0,3^{\mathrm{a}}$ & $0,24 \pm 0,2$ \\
McIntosh, D & $0,54 \pm 0,1^{\mathrm{a}}$ & $0,63 \pm 0,2^{\mathrm{a}}$ & $0,55 \pm 0,2^{\mathrm{a}}$ & $0,56 \pm 0,2$ \\
Shannon-Wiener, H' & $1,8 \pm 0,4^{\mathrm{a}}$ & $2,2 \pm 0,6^{\mathrm{a}}$ & $1,7 \pm 0,7^{\mathrm{a}}$ & $1,85 \pm 0,6$ \\
Riqueza específica de rotíferos & $3,7 \pm 2,4^{\mathrm{a}}$ & $4,0 \pm 2,6^{\mathrm{a}}$ & $2,9 \pm 2,0^{\mathrm{a}}$ & $3,4 \pm 2,2$ \\
Riqueza específica total & $11,1 \pm 4,7^{\mathrm{a}}$ & $13,5 \pm 5,5^{\mathrm{a}}$ & $9,2 \pm 4,6^{\mathrm{a}}$ & $10,7 \pm 4,8$ \\
Margalef, Dmg & $2,1 \pm 0,9^{\mathrm{a}}$ & $2,3 \pm 1,0^{\mathrm{a}}$ & $1,7 \pm 0,9^{\mathrm{a}}$ & $2,0 \pm 0,9$ \\
Menhinick, DMn & $1,01 \pm 0,6^{\mathrm{a}}$ & $0,85 \pm 0,4^{\mathrm{a}}$ & $0,95 \pm 0,4^{\mathrm{a}}$ & $0,95 \pm 0,4$ \\
$\mathrm{~N}^{\circ}$ total de organismos L-1 & $436 \pm 594^{\mathrm{a}}$ & $271 \pm 54^{\mathrm{a}}$ & $329 \pm 656^{\mathrm{a}}$ & $358 \pm 548$ \\
\hline$\overline{\mathrm{X}}$ & & & &
\end{tabular}

$\overline{\mathrm{X}}=$ Promedio. $\mathrm{DE}=$ Desviación estándar.

Letras minúsculas iguales en sentido horizontal indican que los promedios son estadísticamente iguales.

TABLA V. Resumen del análisis de componentes principales (CP) de los Índices bióticos de diversidad alfa $(\alpha)$.

TABLE V. Summary of principal component analysis (APC) for biotic indexes of alpha diversity $(\alpha)$.

\begin{tabular}{lrrr}
\hline Índices & \multicolumn{1}{c}{ CP1 } & \multicolumn{1}{c}{ CP2 } & \multicolumn{1}{c}{ CP3 } \\
\hline Eigenvalue & 6,090 & 1,948 & 1,381 \\
\% de la varianza & 45,587 & 29,221 & 19,383 \\
\hline Pielou, J' & $\mathbf{0 , 9 7 2}$ & 0,109 & 0,026 \\
Berger-Parker, d & $\mathbf{- 0 , 9 6 3}$ & $-0,200$ & 0,001 \\
Simpson, C & $\mathbf{- 0 , 9 4 8}$ & $-0,291$ & 0,020 \\
McIntosh, D & $\mathbf{0 , 9 0 6}$ & 0,331 & 0,035 \\
Shannon-Wiener, H' & $\mathbf{0 , 7 9 8}$ & 0,587 & 0,028 \\
Riqueza específica de rotíferos & 0,180 & $\mathbf{0 , 9 3 0}$ & $-0,168$ \\
Riqueza específica total & 0,339 & $\mathbf{0 , 9 1 5}$ & 0,099 \\
Margalef, Dmg & 0,408 & $\mathbf{0 , 7 5 6}$ & 0,491 \\
Menhinick, Dmn & 0,080 & 0,188 & $\mathbf{0 , 9 1 2}$ \\
$\mathrm{N}^{\circ}$ total de organismos $\mathrm{L}^{-1}$ & 0,100 & 0,148 & $\mathbf{- 0 , 9 0 8}$ \\
\hline
\end{tabular}

Los valores más altos en cada componente para cada variable son mostrados en negrita. 
No se encontró correlación significativa entre la temperatura del agua, temperatura del aire y el oxígeno disuelto con relación a cuatro índices bióticos: Pielou, Shannon-Wiener, riqueza específica total y $\mathrm{N}^{\circ}$ total de organismos $\mathrm{L}^{-1}(\mathrm{r}= \pm 0,01$ a 0,$98 ; \mathrm{P}=0,11$ a 0,98$)$.

Al graficar el número de taxa nuevos que aparecen a lo largo de los 21 puntos de colecta durante el 2004 y el 2005, se observa que en junio del 2004 se registraron 31 taxa, luego en octubre de 2004, se encontraron 8 nuevos taxa incrementándose hasta 39 y finalmente en abril de 2005 se registraron 5 nuevos taxa hasta 44 (Fig. 4). La estabilización del número de taxa $(n=44)$ se da en el muestreo $\mathrm{N}^{\circ} 18$. Sin embargo, los estimadores no paramétricos Chao-2, Jacknife de primer orden y Jacknife de segundo orden indicaron que aun se requiere aumentar el esfuerzo de muestreo, pues indicaron valores de 82, 58 y 69 taxa zooplanctónicas totales como las estimadas para Pantanos de Villa. Esto es debido a que 16 taxa sólo se presentaron en uno de los 21 muestreos y 16 taxa se presentaron entre dos a cinco puntos de muestreo (Fig. 5). El mismo patrón se observó para los rotíferos, pues los estimadores no paramétricos Chao-2, Jacknife de primer orden y Jacknife de segundo orden indicaron valores de 17,23 y 28. La estabilización del número de taxa de rotíferos se da en el muestreo $\mathrm{N}^{\circ} 14$ (Fig. 4). Para los cladóceros, los estimadores no paramétricos Chao-2, Jacknife de primer orden y Jacknife de segundo orden indicaron valores de 15, 11 y 14. La estabilización del número de taxas de cladóceros se da en el muestreo $\mathrm{N}^{\circ} 16$ (Fig. 4). Finalmente, para la fauna acompañante los protistas, los estimadores no paramétricos Chao-2, Jacknife de primer orden y Jacknife de segundo orden indicaron valores de 8, 9 y 10. La estabilización del número de taxa de protistas se da en el muestreo $\mathrm{N}^{\circ} 18$ (Fig. 4). El número de taxa nuevos estuvo correlacionado positivamente con el número de taxa nuevos de rotíferos ( $\mathrm{r}=0,98 ; \mathrm{P}<0,001)$; el número de taxa nuevos estuvo correlacionado con el número de taxa nuevos de cladóceros ( $\mathrm{r}=0,98$; $\mathrm{P}<0,001)$; el número de taxa nuevos estuvo correlacionado con el número de taxa nuevos de protistas ( $\mathrm{r}=0,79 ; \mathrm{P}<0,001)$; el número de taxa nuevos de rotíferos estuvo correlacionado con el número de taxa nuevos de cladóceros $(r=0,96 ; \mathrm{P}<0,001)$, el número de taxa nuevos de rotíferos estuvo correlacionado con el número de taxa nuevos de protistas $(\mathrm{r}=0,71 ; \mathrm{P}<0,001)$, y el número de taxa nuevos de cladóceros estuvo correlacionado con el número de taxa nuevos de protistas $(\mathrm{r}=0,76 ; \mathrm{P}<0,001)$ (Fig. 4).

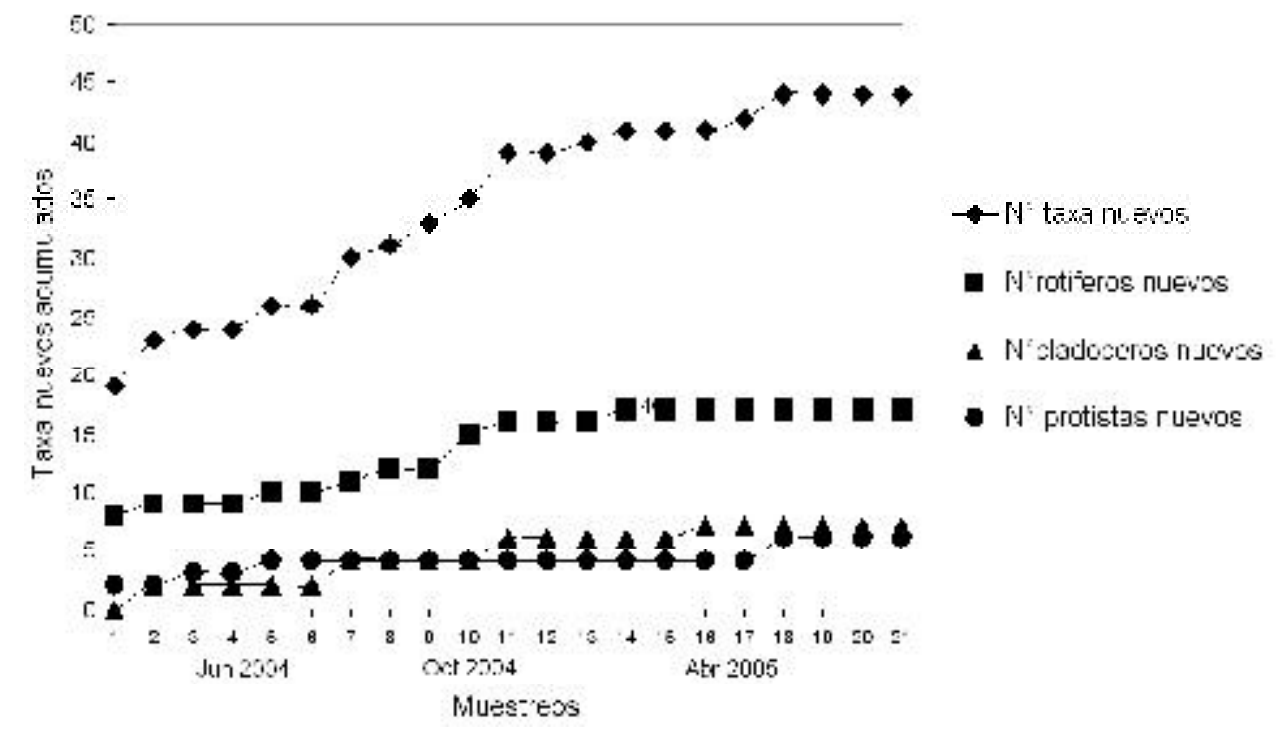

FIGURA 4. Número de taxa nuevos totales, rotíferos nuevos, cladóceros nuevos y protistas nuevos que aparecen a lo largo de los 21 muestreos en los Pantanos de Villa durante el 2004 y el 2005.

FIGURE 4. Number of new total taxa, new rotifers, new cladoceran and new protist that appear along 21 sampling sites in Pantanos de Villa during 2004 and 2005. 


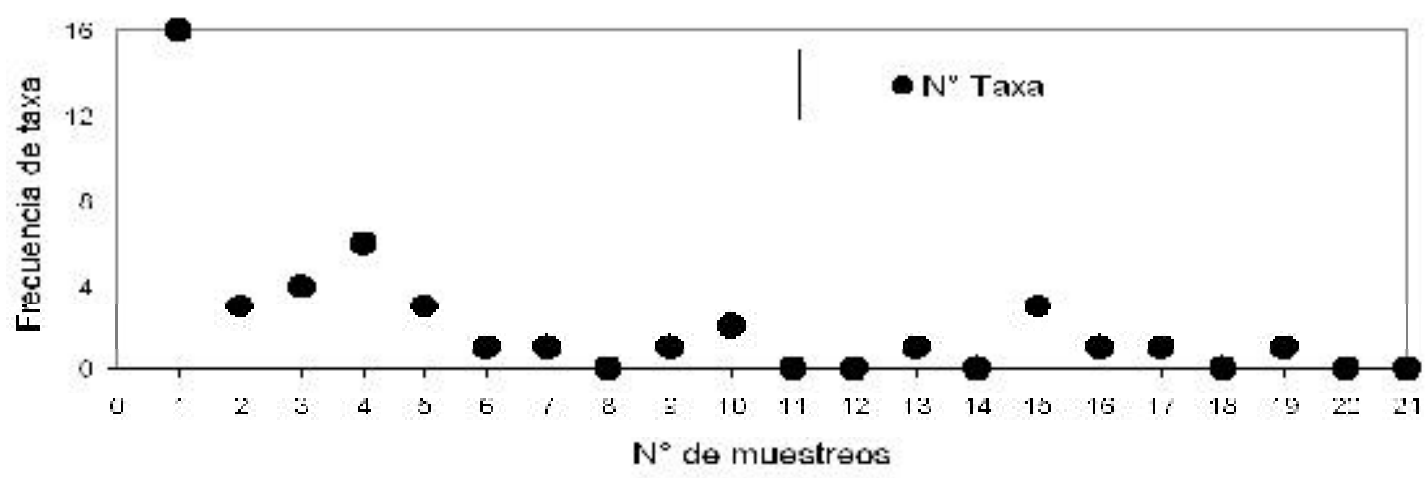

FiguRA 5. Relación entre la frecuencia de taxa con el número de muestreos en los Pantanos de Villa durante el 2004 y el 2005.

FIGURE 5. Relation between frequency of taxa with number of sampling sites in Pantanos de Villa during 2004 and 2005.

DIVERSIDAD BETA

Los cuatro indices de similaridad cualitativos [Sörensen $\left(\mathrm{I}_{\mathrm{S}}\right)$, Jaccard $\left(\mathrm{I}_{\mathrm{J}}\right)$, Sokal y Sneath $\left(\mathrm{I}_{\mathrm{S}-\mathrm{S}}\right)$ y Ochiai-Barkman $\left(\mathrm{I}_{\mathrm{O}-\mathrm{B}}\right)$ ] presentaron el mismo patrón, mayores similaridades en orden descendente para las comunidades zooplanctónicas: octubre 2004abril 2005, junio 2004-abril 2005 y junio 2004-octubre 2005. La presencia o ausencia de los protistas Peridinium sp., Paramecium aurelia, del gastrotrico; los rotíferos $F$. longiseta, Keratella cochleraris, Lecane leontina, Lecane luna y Lecane ludwigi, el molusco Drepanotrema kermatoides, los cladóceros Alona rectangula y Alonella dentifera, y el copépodo Thermocyclops decipiens serían los principales taxa no compartidos entre muestreos (Tabla I). En cambio para los dos índices de similaridad cuantitativos [Sörensen cuantitativo (IS cuantitativo ), Morisita-Horn $\left(\mathrm{I}_{\mathrm{M}-\mathrm{H}}\right)$ ] se observó un patrón opuesto en orden descendente: octubre 2004-junio 2004, junio 2004-abril 2005 y finalmente octubre 2004-abril 2005 (Tabla VI). Trece taxa fueron compartidos en las tres ocasiones de muestreo, destacándose por su abundancia cinco: $R$. terrestres, $B$. calyciflorus, E. sensa, $M$. longisetus y $V$. campanula (Tabla I).

Se observó una correlación lineal significativa en el $\mathrm{N}^{\circ}$ org $\mathrm{L}^{-1}$ de cada taxa entre junio 2004- octubre 2004 ( $\mathrm{r}=0,97 ; \mathrm{P}=0,001$ ), entre junio 2004-abril 2005 $(\mathrm{r}=0,91 ; \mathrm{P}=0,011)$ y entre octubre 2004- abril 2005 $(\mathrm{r}=0,97 ; \mathrm{P}=0,001)$. El índice de beta- 2 mostró para junio de 2004, octubre de 2004 y abril de 2005 valores de 53,69 \% (24,60-100), 75,88 \% (51,85-100) y $37,72 \%(0-58,3)$, respectivamente.

\section{DISCUSION}

Los principales grupos que componen las comunidades zooplanctónicas son los protistas, rotíferos y crustáceos, particularmente copépodos y cladóceros (Sampaio et al. 2002, Neves et al. 2003, Keppeler \& Hardy 2004a).

Los rotíferos fueron el grupo con mayor riqueza taxonómica y abundancia durante los tres periodos de muestreo (Tabla I). Sampaio et al. (2002) evaluó la composición y abundancia de la comunidad zooplanctónica conformada por rotíferos, cladóceros y copepódos en siete reservorios del río Paranapanema, Brasil durante cuatro periodos del año. En cambio, Neves et al. (2003) analizó la composición taxonómica de dos grupos del zooplancton: rotíferos y cladóceros, en los márgenes del río Cuiabá, Mato Grosso, Brasil. En estos dos últimos estudios se encontraron resultados similares al indicar que los rotíferos fueron los dominantes sobre los otros representantes del zooplancton, semejante a lo encontrado en el presente estudio (Tabla I). Este patrón de mayor riqueza taxonómica y abundancia de los rotíferos es el común en ambientes dulceacuícolas tropicales, sean estos lagos, lagunas, reservorios, ríos o arroyos. Esto puede ser atribuido a que estos organismos son estrategas $r$, oportunistas, tamaño pequeño, ciclo de vida corto y amplia tolerancia a una variedad de factores ambientales (Neves et al. 2003). De las 17 especies de rotíferos 7 se presentaron en forma constante en las tres ocasiones de muestreo (Tabla I). En cambio, 6 especies 
forman parte de una sucesión de especies de rotíferos, pues se presentaron sólo en una de las ocasiones de los tres muestreos (Tabla I). En adición, el amplio espectro de partículas alimenticias explotadas por los rotíferos, el cual tiene la habilidad de consumir bacterias, algas y detritus de diferentes tamaños, permite diferentes dietas para muchas especies simultáneamente presentes en los cuerpos de agua (Arora \& Mehra 2003). La zona litoral ha sido señalada como la que presenta una mayor diversidad de rotíferos en comparación a la zona limnética (Rodriguez \& Matsumura-Tundisi 2000). Se encontró en el presente estudio una dominancia numérica de los rotíferos a pesar de emplear una malla de 75 $u \mathrm{~m}$, el cual podría subestimar la cantidad de pequeños organismos. Dos especies de rotíferos E. sensa y B. calyciflorus fueron las más abundantes durante todo el muestreo (Tabla I). Este patrón está en oposición a lo encontrado por Sampaio et al. (2002), quienes a pesar de evaluar comunidades zooplanctónicas en reservorios dulceacuícolas, observaron que diferentes especies de rotíferos dominan en diferentes periodos de muestreo y no hay una sola especie dominante durante todo el año. Brachionus calyciflorus es una especie indicadora de eutroficación, observándose altas densidades de este rotífero durante las tres ocasiones de muestreo, lo cual está en armonía con niveles de oxígeno disuelto bajos, cuyo promedio fue menor a $4 \mathrm{mg} \mathrm{L}^{-1}$ en el presente estudio (Tabla II) (Sampaio et al. 2002). Las familias Lecanidae, típicamente litoral con cuatro taxa y Brachionidae, típicamente planctónica con cinco taxa fueron las familias de rotíferos más frecuentemente observadas en el presente trabajo (Tabla I), las cuales son predominantes en ambientes tropicales (Keppeler \& Hardy 2004b).

En los copépodos, la predominancia numérica de estadios tempranos de desarrollo, naupliosmetanauplios es el patrón más común, registrándose en diferentes hábitats dulceacuícolas como los Humedales de Ventanilla, Callao, Perú (Moreno 2003), y también encontrado en el presente estudio, con 25, 24 y 31 org L $\mathrm{L}^{-1}$ para junio del 2004, octubre del 2004 y abril del 2005, respectivamente. Las altas densidades de formas inmaduras son resultados de una continua reproducción de estos organismos, en las regiones neotropicales, con superposición de varias cohortes. Según Neves et al. (2003), un factor que puede determinar la proporción de formas inmaduras y de adultos es la intensidad de depredación y el balance de depredación por invertebrados y vertebrados. La existencia de formas inmaduras es de gran importancia para la estructura comunitaria del zooplancton, con relación a la dinámica poblacional, y también a los aspectos tróficos, debido a que las fases tempranas pueden ocupar nichos diferentes que los adultos. Así los nauplios de ciclopoideos son predominantemente filtradores herbívoros y los adultos tienen hábitos de alimentación raptorial, son predominantemente carnívoros (Neves et al. 2003). La proporción de naupliometanauplio y ciclopoideos adultos fluctuó de 1,04, 2,18 y 2,58 para junio 2004, octubre 2004 y abril 2005 , respectivamente. A pesar que la dieta de los copépodos ciclopoideos que tienden a ser omnívoros al alimentarse de plancton y de otros microinvertebrados bénticos y su amplitud de nicho les favorecen. Sin embargo, su reproducción obligatoria sexual y ciclos de vida más largos, resultan en un reducido número de generaciones en comparación con los rotíferos y los cladóceros (Sampaio et al. 2002). La especie cosmopolita y pantropical Thermocyclops decipiens (Kiefer, 1929), muy común en ambientes dulceacuícolas de Brasil en altas densidades y considerada una especie típica de ambientes disturbados y enriquecido por nutrientes (Suárez-Morales et al. 2004), en el presente estudio fue considerada especie rara (Tabla I). De acuerdo con Sampaio et al. (2002), las especies de Thermocyclops son consideradas generalmente herbívoras o algunas veces carnívoras, mientras que las especies tropicales de Mesocyclops son consideradas carnívoras o detritívoras (Suárez-Morales et al. 2004). A pesar de que los copépodos calanoideos son muy comunes en los ambientes dulceacuícolas tropicales, éstos no se han registrado en el presente estudio (Tabla I).

Los cladóceros han sido señalados como buenos indicadores del estado trófico en ecosistemas lénticos. Inclusive el rango del tamaño de las especies es usado como un indicador de calidad del agua. Las especies de cladóceros grandes, principalmente Daphniidae, están relacionadas con una mejor calidad del agua, por el control del fitoplancton por herbivoría; en cambio la predominancia de pequeños cladóceros está relacionada con aguas más eutróficas (Santos-Wisniewski et al. 2002). En el presente estudio predominaron las especies pequeñas de cladóceros como el chydorido Alona 
cambouei Guerne \& Richard, 1893, relacionada con tenores de oxígeno bajos (Tablas I y II), y de ser habitantes típicos de la zona litoral (SantosWisniewski et al. 2002). Por otro lado, Gilbert (1988) propone que la depredación por los grandes cladóceros tiene un rol modelador y de control en las comunidades naturales del zooplancton, principalmente sobre los rotíferos; así los rotíferos pueden ocurrir en altas densidades con la presencia de pequeños cladóceros, en cambio los rotíferos no son componentes abundantes del zooplancton en la presencia de cladóceros grandes (> 1,2 $\mathrm{mm}$ ). En el presente estudio, las especies pequeñas de cladóceros y las especies de rotíferos predominaron y coexistieron en los Pantanos de Villa. En cambio, los cladóceros grandes no fueron dominantes (Tabla I). DeMott et al. (2001) indica que los cladóceros grandes pueden ser excluidos por la interferencia de filamentos de cianobacterias. Se han reportado hasta la fecha 171 especies de protistas en los Pantanos de Villa (Guillén et al. 2003). Sin embargo, debido al protocolo empleado relacionado con 1) mallas planctónicas de 75 $u \mathrm{~m}, \mathrm{y}$ 2) fijador y preservante a base formol azucarado al $10 \%$, sólo se registraron seis especies de protozoos, lo cual es una subestimación de la diversidad del grupo (Tabla I). Esto explicaría por qué no se encontró una correlación positiva y significativa entre el número de taxa totales por punto de muestreo y el número de taxa de protistas. La presencia del ciliado Vorticella estaría indicando la existencia de aguas con alta presencia de materia orgánica (Guillén et al. 2003).

Rhabdolaimus terrestris es un nematodo bacterívoro muy abundante en los ambientes dulceacuícolas y terrestres (Ettema et al. 1998). En el presente trabajo fue considerada una especie constante (Tabla I).

No se observó diferencias entre los diez diferentes índices bióticos de diversidad alfa en los Pantanos de Villa relacionados a la estacionalidad de los tres periodos de muestreo (Tabla IV), a pesar que existieron diferencias en la temperatura del agua, del aire y el OD entre los periodos de muestreo (Tabla II), fluctuación en el ciclo hidrológico de los Pantanos de Villa con incremento en el nivel durante el invierno, y un número de puntos de muestreo menor en octubre de 2004 en comparación a junio de 2004 y abril de 2005. La diversidad beta de similaridad entre los tres pe- riodos de muestreo indicó valores muy cercanos entre sí, no fluctuando más del $8 \%$ entre los cuatro índices cualitativos y del 19\% entre los dos cuantitativos (Tabla VI). Diferencias estacionales en las comunidades planctónicas han sido observadas en diversos cuerpos de agua dulceacuícolas de la región neotropical (Sampaio et al. 2002, Aoyagui et al. 2003, Neves et al. 2003, Keppeler \& Hardy 2004b).

La diversidad de especies zooplanctónicas tiende a ser baja en ecosistemas controlados por variables físicas y químicas y alta en ecosistemas controlados biológicamente (Keppeler \& Hardy 2004b). La ausencia de estacionalidad de los índices bióticos de diversidad alfa, a pesar de la fluctuación de la temperatura y del oxígeno entre los tres periodos de muestreo; así como la falta de correlación con los principales indicadores bióticos, pudiera aparentemente mostrar que el control del ecosistema es biológico más que físico y químico. Sin embargo, al no registrarse en el presente estudio a los depredadores del zooplancton; así como evaluar el pH y la conductividad sólo en la última ocasión de muestreo, y amplio distanciamiento en el tiempo de las ocasiones de muestreo, no nos permiten tener resultados concluyentes. En adición, la perturbación antrópica del humedal sería un factor de influencia en la diversidad del zooplancton (Keppeler \& Hardy 2004b). La baja profundidad de un cuerpo de agua es un factor que disminuye la diversidad zooplanctónica en un hábitat (Keppeler \& Hardy 2004a). El cuerpo de agua estudiado tiene una profundidad promedio de $50,5 \mathrm{~cm}$, siendo un factor más que contribuye a su alta diversidad (Tabla II).

Allen et al. (1999) encontró baja congruencia en la riqueza de especies de varios grupos de organismos lénticos como diatomeas, rotíferos, crustáceos, macroinvertebrados, peces y aves. En el presente estudio, se observó un patrón opuesto, una correlación positiva entre la riqueza total de taxa y la riqueza de rotíferos; entre la riqueza total de taxa y la riqueza de copépodos, y entre la riqueza total de taxa y la riqueza de cladóceros. Los rotíferos, al presentar la mayor riqueza y abundancia en el humedal de Pantanos de Villa y la mayor correlación con la riqueza total de taxa, se convierten en los más efectivos taxa "sombrilla" de las comunidades zooplanctónicas con fines de conservación de este ecosistema (Allen et al. 1999). 
Los presentes resultados indican la necesidad de continuar evaluando este humedal y de conservar este cuerpo de agua inmerso dentro del ecosistema urbano de Lima, Perú, el cual contie- ne una diversidad y abundancia significativa de invertebrados acuáticos, muchos de los cuales no han sido registrados previamente para este ecosistema.

TABLA VI. Diversidad beta de similaridad empleando seis índices entre los tres periodos de muestreo (junio 2004, octubre 2004 y abril 2005) para los Pantanos de Villa.

TABLE VI. Beta diversity of similarity employing six indexes between three periods of survey (June 2004, October 2004 and April 2005) for Pantanos de Villa.

\begin{tabular}{|c|c|c|c|c|}
\hline & \multicolumn{4}{|c|}{ Indice de Sörensen $\left(\mathrm{I}_{s}\right)$} \\
\hline & & Junio 2004 & Octubre 2004 & Abril 2005 \\
\hline Indice de & Junio 2004 & - & 0,64 & 0,67 \\
\hline \multirow[t]{4}{*}{ Jacard $\left(\mathrm{I}_{\mathrm{J}}\right)$} & Octubre 2004 & 0,48 & - & 0,71 \\
\hline & Abril 2005 & 0,51 & 0,55 & - \\
\hline & \multicolumn{4}{|c|}{ Indice de Sokal y Sneath $\left(\mathrm{I}_{\mathrm{ss}}\right)$} \\
\hline & & Junio 2004 & Octubre 2004 & Abril 2005 \\
\hline Indice de & Junio 2004 & - & 0,32 & 0,35 \\
\hline Ochiai- & Octubre 2004 & 0,022 & - & 0,40 \\
\hline \multirow[t]{3}{*}{$\operatorname{Barkman}\left(\mathrm{I}_{\mathrm{O}-\mathrm{B}}\right)$} & Abril 2005 & 0,025 & 0,026 & - \\
\hline & \multicolumn{4}{|c|}{$\begin{array}{l}\text { Indice de Sörensen cuantitativo } \\
(\text { IS }\end{array}$} \\
\hline & & Junio 2004 & Octubre 2004 & Abril 2005 \\
\hline Indice de & Junio 2004 & - & 0,29 & 0,28 \\
\hline Morisita- & Octubre 2004 & 0,79 & - & 0,25 \\
\hline Horn $\left(\mathrm{I}_{\mathrm{M}-\mathrm{H}}\right)$ & Abril 2005 & 0,66 & 0,58 & - \\
\hline
\end{tabular}

\section{AGRADECIMIENTOS}

Se agradece a los estudiantes del curso de Biología de la Conservación de los semestres 2004-I, 2004II y 2005-I de la Facultad de Ciencias Biológicas de la Universidad Ricardo Palma, por su colaboración en la toma de las muestras de agua en el campo.

\section{BIBLIOGRAFIA}

Allen, A.P., T.R. Whittier, D.P Larsen, P.R. Kaufmann, R.J. O’Connors \& R.M. Hughes. 1999. Concordance of taxonomic richness patterns across multiple assemblages in lake of the northeastern
United States. Canadian Journal of Fisheries and Aquatic Sciences 56: 739-747.

Alvariño, L. F. 1990. Fauna Cladócera (Crustácea) del Lago Junín. Tesis para optar al Título de Licenciatura en Biología. Universidad Ricardo Palma. Lima, Perú. 169 pp + Anexos.

Amaya, P., C. Muñoz, \& R. Cueva. 1999. Cálculo de los beneficios económicos para mejorar la calidad del agua del humedal "Los Pantanos de Villa". Wichay Yachay 3(2): 82-91.

Aoyagui, A.S.M., C.C. Bonecker, F.A. Lansac-TôHa \& L.F.M. Velho. 2003. Structure and dynamics of rotifers in Corumbá reservoir, State of GóiasBrazil. Acta Scientiarum 25: 31-39.

APHA. 1995. Standard Methods for the Examination of Water and Wastewater. Public HealthAssoc. Washington. $19^{\text {th }}$ Ed. 
Arora, J. \& N.K. M ehra. 2003. Species diversity of planktonic and epiphytic rotifers in the backwaters of the Delhi segment of the Yamuna Rivers, with remarks on new records from India. Zoological studies 42: 239-247.

Bini, L.M., C.C. Bonecker \& F.A. Lansac-T ÔHa. 2001. Vertical distribution of rotifers on the upper Paraná River floodplain: the role of thermal stratification and chlorophyll-a. Studies on Neotropical Fauna and Environment 36: 241246.

BLEDZKI, L.A. \& A.M. ElLISON. 2003. Diversity of rotifers from northeastern U.S.A. bogs with new species records for North America and New England. Hydrobiologia 497: 53-62.

Del Río, M. \& R. Valdivia. 1989. Copépodos dulceacuícolas de vida libre en el Perú. Familia Cyclopidae. Orden Cyclopoida. Boletín de Lima 63: $71-80$

De Meester, L.D., S. Declerck, R. Stoks, G. Louette, F. Van de Meutter, T. De Bie, E. Michels \& L. BRENDONCK, L. 2005. Ponds and pools as model systems in conservation biology, ecology and evolutionary biology. Aquatic Conservation: Marine and Freshwater Ecosystems 15: 715-725.

DeMott, W., R. Gulati \& E. Van Donk. 2001. Daphnia food limitation in three hypereutrophic Dutch lakes: evidence for exclusion of large-bodied species by interfering filaments of cyanobacteria. Limnology and Oceanography 46: 2054-2060.

Duarez, J. 1998. Composición y riqueza de arañas (Arácnida: Araneae) en los Pantanos de Villa. En: Pantanos de Villa. Biología y Conservación. pp. 105-113. (eds. Cano, A. \& K. R. Young). Museo de Historia Natural - UNMSM. Lima.

Ettema, C.H., D.C. Coleman, G. Vellidis, R. Lowrance \& S.L. Rathbun. 1998. Spatiotemporal distributions of bacterivorous nematodes and soil resources in a restored riparian wetland. Ecology 79: 2721-2734.

García, A.I., G.R. Vivar, M.J. Quezada, \& P.M. Huamán. 1996. Insectos acuáticos biorreguladores de larvas de mosquito presentes en los "Pantanos de Villa", Lima, Perú. Revista Cubana de Medicina Tropical 48: 227-228.

Gilbert, J. J. 1988. Supression of rotifer populations by Daphnia: A review of the evidence, the mechanisms, and the effects on zooplancton community structure. Limnology and Oceanography 33: 1286-1303.

Grosspietsh, W. J. 1999. Estudio preliminar del zooplancton peruano. Biota 99: 67-71.

Guillen, C. \& J. Barrio. 1995. Los Pantanos de Villa y sus aves. Boletín de Lima 91-96: 53-58.

Guillén, G. \& E. Morales. 2003. Primeros registros de helmintos parásitos en Fulica ardesiaca (Aves: Rallidae): Pantanos de Villa-Lima. Revista Peruana de Biología 10:203-208.

Guillén, G., E. Morales \& R. Severino. 2003. Adiciones a la fauna de protozoarios de los Pantanos de Villa, Lima, Perú. Revista Peruana de Biología 10: 175-182.

Harrinson, S.S., S.J. Ross \& J.H. Lawton. 1992. Beta diversity on geographic gradients in Britain. Journal of Animal Ecology 61: 151-158.

Holynska, M., J.W. Reid \& H. Ueda. 2003. Genus Mesocyclops Sars, 1914. Copepoda: Cyclopoida genera Mesocyclops and Thermocyclops (ed. By H. Ueda and J.W. Reid), pp. 12-213. Guides to the Identification of the Microinvertebrates of the Continental Waters of the World. Backhuys Publishers, Amsterdam.

Iannacone, J., J. Mansilla. \& K. Ventura, K. 2003. Macroinvertebrados en las lagunas de Puerto Viejo, Lima-Perú. Ecología Aplicada 2: 116-124.

José de PAGGI, S. 1989. Rotíferos de algunas provincias del noroeste argentino. Revista de Hidrobiología Tropical 22: 223-238.

Keppeler, E.C. \& E.R. Hardy. 2004a. Vertical distribution of zooplankton in the water column of Lago Amapá, Rio Branco, Acre, Brazil. Revista Brasileira de Zoologia 21: 169-177.

Keppeler, E.C. \& E.R. Hardy. 2004b. Abundance and composition of rotifera in an abandoned meander lake (Lago Amapá) in rio Branco, Acre, Brazil. Revista Brasileira de Zoologia 21: 233-241.

Koste, W. 1972. Rotatorien aus Gewassen Amazoniens. Amazoniana 3: 258-505.

Koste, W. 1978. Rotatoria. Die Rädertiere Mittleeuropas. Vol. 2. Stuttgart: Gebrüder Bomtraeger. Berlin. $673 \mathrm{p}$.

León, B., A. CANo \& K. Young. 1995. La flora vascular de los Pantanos de Villa, Lima, Perú: Adiciones y guía para las especies comunes. Publicaciones del Museo de Historia Natural-UNMSM. (B) 38:139.

Loвo, E. \& G. Leighton. 1986. Estructuras comunitarias de las fitocenosis planctónicas de los sistemas de desembocaduras de ríos y esteros de la zona central de Chile. Revista de Biología Marina 22: $1-29$.

MedRI I.M. \& Lopes J. 2001. Coleopterofauna em floresta e pastagem no norte do Paraná, Brasil, colectada con armadilha de solo. Revista Brasileira de Zoologia 18: 125-133.

Mirabdullayev, I.M., J.W. Reid \& H. Ueda. 2003. Genus Thermocyclops Kiefer, 1927. Copepoda: Cyclopoida genera Mesocyclops and Thermocyclops. En: Guides to the Identification of the Microinvertebrates of the Continental Waters of the World. (Eds. Ueda, H. \& Reid, J.W.), pp. 214-302. Backhuys Publishers, Amsterdam.

Modenutti, B.E. 1998. Planktonic rotifers of Samboronbón River Basin (Argentina). Hydrobiologia 387/388: 259-265.

Montoya, H. 1984. Algas de la Laguna de Villa (Lima): Cyanophyta, Chlorophyta y Chrysophyta (Xanthophyceae). Boletín de Lima 31:49-62, 75-89.

Moreno, A.R.P. 2003. Comunidades Hidrobiológicas en los Humedales de Ventanilla, Callao. Tesis de Licen- 
ciatura. Universidad Nacional Federico Villarreal. Lima, Perú. 67 pp. + Anexos.

Moreno, C.E. 2001. Métodos para medir la biodiversidad. Manuales y Tesis SEA. Sociedad Entomológica Aragonesa Ed. Madrid, España. 80 pp.

Nagorskaya, L. 2004. Living freshwater ostracoda (Crustacea) of a floodplain relict oak forest (Polesye, Belarus). Revista Española de Micropaleontología 36: 135-145.

Neves, I.F., O. Rocha, K.F. Roche \& A.A. Pinto. 2003. Zooplankton community structure of two marginal lakes of the River Cuiabá (Mato Grosso, Brazil) with analysis of rotifera and cladocera diversity. Brazilian Journal of Biology 63: 329343.

Paraense, W.L. 2003. Planorbidae, Lymnaeidae and Physidae of Peru (Mollusca: Basommatophora). Memorias Instituto Oswaldo Cruz 98: 767-771.

Ramos, R., J. Carvajal, M. Carter \& L. Zúñiga. 2004. Patrones de migración vertical de tres poblaciones de zooplancton en un lago chileno. Revista Chilena de Historia Natural 77:29-41.

Rejas, D., S. Declerck, J. Auwerkerken, P. Tak \& L. De Meester. 2005. Plankton dynamics in a tropical floodplain lake: fish, nutrients, and the relative importance of bottom-up to top-down control. Freshwater Biology 50: 52-69.

Riofrío, J., I. SAMANEZ \& F. CARRASCo. 2003. Limnological characteristics of the Amazonic Cashibococha Lake (Ucayali-Perú) at 2001. Revista Peruana de Biología 8: 183-194.

Rodriguez, M.P. \& T. M atsumura-Tundisi. 2000. Variation of density, species composition and dominance of rotifers at a shallow tropical reservoir (Broa Reservoir, SP, Brazil) in a short scale time. Revista Brasileira de Biologia 60: 1-9.

Sampaio, E.V., O. Rocha, T. Matsumura-Tundisi \& J.G. Tundisi. 2002. Composition and abundance of zooplancton in the limnetic zone of seven reservoirs of the Paranapema River, Brazil. Brazilian Journal of Biology 62: 525-545.

Santos-Wisniewski, M.J., O. Rocha, A.M. Güntzel \& T. M atsumura-Tundisi. 2002. Cladocera Chydoridae of high altitude water bodies (Serra da Mantiqueira), in Brazil. Brazilian Journal of Biology 62: 681-687.

Sarmiento, L. \& E. Morales. 1998. Protozoarios, Turbelarios y Nematodos de los Pantanos de Villa, Lima, Perú: Su importancia en el ecosistema. En: Pantanos de Villa. Biología y Conservación. (Eds. Cano, A. \& K. R. Young). pp. 41-54. Museo de Historia Natural - UNMSM. Lima.
Segers, H. 1995. Rotifera 2: The Lecanidae (Monogononta). En: Guides to the identification of the microinvertebrates of the continental waters of the world 6. (Eds. H.J. Dumont, T. Nogrady). The Hagues: SPB Academic Publishing. The Netherlands. 226 pp.

Suárez-Morales, E., J.W. Reid, F. Fiers \& T.M. Iliffe. 2004. Historical biogeography ad distribution of the freshwater cyclopine copepods (Copepoda, Cyclopopida, Cyclopinae) of the Yucatan Peninsula, Mexico. Journal of Biogeography 31: 1051-1063.

Suárez-Morales, E., J.W. Reid \& M. Elías-Gutiérrez. 2005. Diversity and distributional patterns of Neotropical freshwater copepods (Calanoida: Diaptomidae). International Review of Hydrobiology 90: 71-83.

Thiengo, S.C., M.A. Fernández, M.F. Boaventura, C.E. Grault, H.F.R. Silva, A.C. Mattos \& S.B. SanTOS. 2001. Freshwater snails and schistosomiasis mansoni in the State of Rio de Janeiro, Brazil: IMetropolitan Mesoregion. Memorias do Instituto Oswaldo Cruz, Rio de Janeiro 96: 177-184.

VALDivia, R. \& L. BuRger. 1990. Observaciones sobre la fauna Cladocera de algunas lagunas altoandinas del Perú. Boletín Lima 71: 63-69.

Valdivia, R. \& F. Zambrano. 1989. Cladóceros de la Laguna de Paca, Junín. Relaciones ecológicas entre hábitat y especie. Boletín de Lima 64: 83-89.

VALQUi, T. 2004. Where to watch birds in Peru. Gráfica Ñañez S.A. Lima, Perú. 382 pp.

Vivanco, M. 1999. Análisis estadístico multivariable. Teoría y práctica. Ed. Universitaria. Santiago de Chile. 234 pp.

Vivar, G. R., M.P. Huamán \& C.H. Larrea. 1994. Clave de identificación para gasterópodos pulmonados dulceacuícolas de importancia médica en el Perú. Biotempo 1: 33-35.

Vivar, G.R., M.R. Ramírez \& P.M. Huamán. 1998. Moluscos de los Pantanos de Villa y su aporte a la conservación. En: Pantanos de Villa. Biología y Conservación. pp. 55-73. (eds. Cano, A. \& K. R. Young). Museo de Historia Natural UNMSM. Lima.

WetZeL, R.G \& G.E. Likens. 2001. Limnological analyses. Springer-Verlag New York. $3^{\text {th }}$ Ed. $429 \mathrm{pp}$

Young, K. 1998. El ecosistema. En: Pantanos de Villa. Biología y Conservación. pp. 3-20. (eds. Cano, A. \& K. R. Young). Museo de Historia Natural UNMSM. Lima.

ZAR, J.H. 1996. Bioestatistical Analysis. $3^{\text {th }}$ Ed. PrenticeHall. Inc. Upper Saddle River, New Jersey. 662 p. 